\title{
Resonantly photopumped lasing and its switching behavior in a photonic crystal nanolaser
}

\author{
Kengo Nozaki, ${ }^{\text {a) }}$ Shota Kita, Yoshiki Arita, and Toshihiko Babab) \\ Department of Electrical and Computer Engineering, Yokohama National University, 79-5 Tokiwadai, \\ Hodogayaku, Yokohama 240-8501, Japan and Core Research for Evolutional Science and Technology \\ (CREST), Japan Science and Technology (JST) Agency, 5 Sanbancho, Chiyodaku, Tokyo 102-0075, \\ Japan
}

(Received 27 November 2007; accepted 14 December 2007; published online 14 January 2008)

\begin{abstract}
A GaInAsP photonic crystal nanolaser was operated by resonant optical pumping in which the effective cavity area was selectively excited. The laser mode intensity was markedly enhanced when the pump wavelength was tuned to a higher-order cavity mode, and room-temperature continuous-wave lasing was obtained in the fundamental mode. Furthermore, the switching behavior due to a modal wavelength shift was clearly observed, suggesting the possibility of bistable operation, and so on. () 2008 American Institute of Physics. [DOI: 10.1063/1.2832670]
\end{abstract}

Photonic crystal (PC) nanolasers could become a key active element in functional integrated photonic chips, because of their small size, low threshold, and strong cavity quantum electrodynamic effects. ${ }^{1-4}$ Recently, we fabricated the smallest possible PC nanolaser, consisting of only the shift of two lattice points (therefore, termed $H 0$ nanolaser) in a GaInAsP PC slab. ${ }^{5}$ In this device, the modal field is localized in an area of only $0.17 \mu \mathrm{m}^{2}=0.82(\lambda / n)^{2}$, where $\lambda$ is the modal vacuum wavelength and $n$ is the refractive index of the slab. We achieved room temperature (RT) continuouswave (cw) operation by optically pumping it at a wavelength of $0.98 \mu \mathrm{m}$ with a pump spot diameter of $2.5 \mu \mathrm{m} .{ }^{6}$ However, this high-frequency pumping with a spot size significantly larger than the modal area leads to unwanted carrier excitation and excess device heating, resulting in strong power saturation and device degradation under the influence

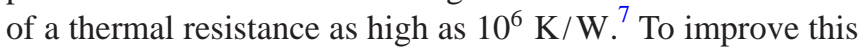
situation, we turned to resonant pumping, adjusting the pump wavelength to a high-order cavity mode. To date, the resulting resonant and off-resonant laser characteristics have only been investigated at low temperatures. ${ }^{8}$ This method effectively pumps only the modal area, thereby suppressing excess heating. In this letter, we demonstrate resonantly pumped RT cw operation of $H 0$ nanolaser. We also observe nonlinear switching behavior in the lasing characteristics, arising from an unusual modal shift.

To fabricate the device, we prepared a GaInAsP-InP epitaxial wafer with a slab active layer consisting of quaternary single-compressively strained quantum well (CS-QW) and separate confinement heterostructure ( $\mathrm{SCH}$ ) layers. The total thickness of the slab was $180 \mathrm{~nm}$. The photoluminescence spectrum of the CS-QW was centered at $1.55 \mu \mathrm{m}$. To this wafer, $H 0$ nanocavity surrounded by 20 rows of $\mathrm{PC}$ airholes in a triangular lattice was formed by e-beam lithography, $\mathrm{HI} / \mathrm{Xe}$ inductively coupled plasma etching, ${ }^{9}$ and $\mathrm{HCl}$ selective wet etching, as shown in Fig. 1(a). The lattice constant $a$, normalized airhole diameter $2 r / a$, and normalized airhole shift $s / a$ were $580 \mathrm{~nm}, 0.52$, and 0.16 , respectively.

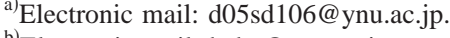

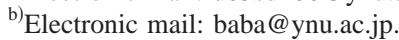

In order to characterize the device, pump light was focused to a $2.5 \mu \mathrm{m}$ spot on the top surface of the nanocavity through an objective lens. The emission from the device was directly collected by a tapered multimode fiber and analyzed by an optical spectrum analyzer (OSA). Figure 1(b) shows the emission spectrum obtained at RT $(293 \mathrm{~K})$ by pulsed photopumping at $\lambda=0.98 \mu \mathrm{m}$ with a duty factor of $0.075 \%$. Two resonant peaks were observed at $\lambda=1537$ and $1584 \mathrm{~nm}$. The broad spectral width of these peaks was caused by a combination of thermally induced wavelength chirping ${ }^{10}$ and the $5 \mathrm{~nm}$ resolution of the OSA. Using three-dimensional finite-difference time-domain (FDTD) calculations, we confirmed that the peaks at 1537 and $1584 \mathrm{~nm}$ correspond to the dipole and monopole modes, respectively, as illustrated in the inset of Fig. 1(b).

In the resonant photopumping experiment, the dipole and monopole modes were used for pumping and lasing, respectively. Figure 2(a) shows the laser mode intensity as a function of the pump wavelength. As the wavelength was tuned to the dipole mode, the laser mode intensity was dramatically enhanced, due to resonant pumping. Figure 2(b) shows the laser mode intensity versus irradiated power. The inset diagram shows the $\mathrm{cw}$ emission spectrum when the

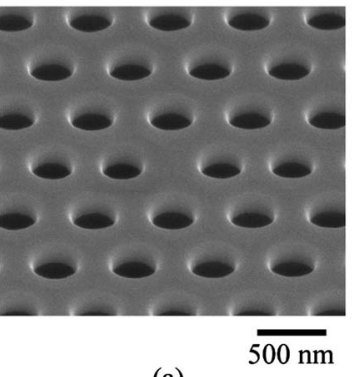

(a)

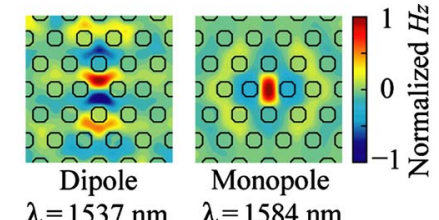

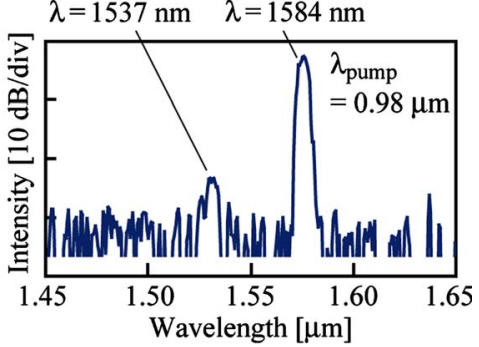

(b)
FIG. 1. (Color online) $H 0$ nanolaser. (a) Scanning electron micrograph of the fabricated device. (b) Resonant modes observed in the photoluminescence spectrum for high-frequency pumping at $\lambda=0.98 \mu \mathrm{m}$. 


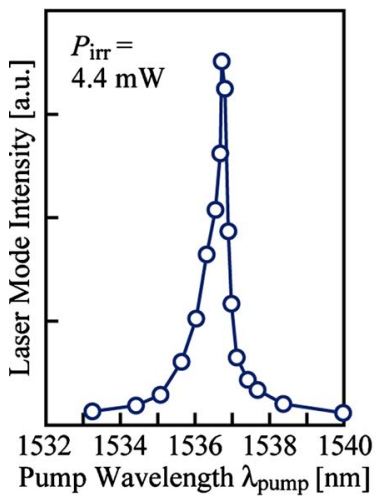

(a)

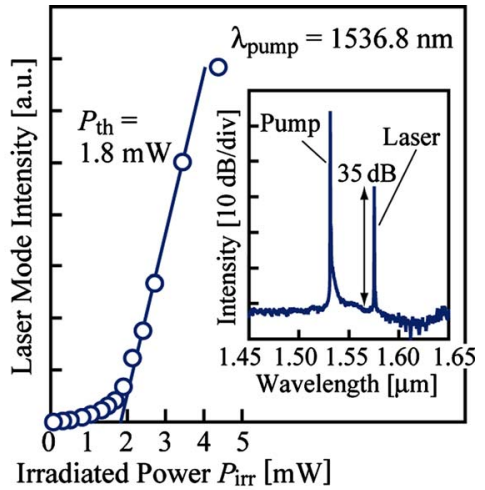

(b)
FIG. 2. (Color online) Laser characteristics obtained by resonantly pumping at $\lambda=1536.8 \mathrm{~nm}$. (a) Laser mode intensity vs pump wavelength. (b) Main graph-laser mode intensity vs irradiated power. Inset figure-lasing spectrum when pumped above threshold.

pump wavelength is fixed at that of the dipole mode at RT. Lasing was confirmed by monitoring the emitted intensity and spectrum. The strongest peak in the inset of Fig. 2(b) is due to pump light reflected from the sample surface and coupled to the detection fiber. The laser mode peak intensity is $35 \mathrm{~dB}$ above the background level. The irradiated threshold power was $1.8 \mathrm{~mW}$, which is 50 times higher than that for pumping at $\lambda=0.98 \mu \mathrm{m}$. In the latter case, the absorption efficiency of the pump light was $\eta_{\mathrm{abs}}=(1-R)\left(1-e^{-\alpha t}\right) /(1$ $\left.-R e^{-\alpha t}\right)=22 \%$, where $R=30 \%$ is the reflectivity at the slab surfaces, $\alpha=20000 \mathrm{~cm}^{-1}$ is the absorption coefficient, and $t=180 \mathrm{~nm}$ (including the SCH layers) is the absorptive layer thickness. ${ }^{11}$ For resonant pumping at $\lambda \approx 1.5 \mu \mathrm{m}$, on the other hand, $\alpha$ decreases to between 3000 and $5000 \mathrm{~cm}^{-1}, 12,13$ and $t$ is only $4 \mathrm{~nm}$ for the CS-QW layer, so that $\eta_{\text {abs }}$ would be estimated to range from merely $0.12 \%$ to $0.20 \%$. But in addition, $\eta_{\text {abs }}$ is enhanced by the cavity $Q$ factor and is reduced by the poor coupling efficiency of the pump light to the cavity mode. The total cavity $Q$ for the dipole mode is approximately $Q_{\text {total }} \approx\left(Q_{a}^{-1}+Q_{\text {ver }}^{-1}+Q_{\text {par }}^{-1}\right)^{-1}$, where the three $Q$ factors on the right-hand side are determined by interband absorption, out-of-plane radiation loss, and parasitic loss, respectively, caused by free-carrier absorption and by light scattering in the fabricated device. We estimate $Q_{a}$ to be 2000 for $\alpha=4000 \mathrm{~cm}^{-1}$ with an optical confinement factor $\Gamma$ into the CS-QW layer of $2 \%$. In our previous study, we also determined that $Q_{\mathrm{ver}}=2500$ and $Q_{\mathrm{par}}=20000$ for the dipole mode, resulting in $Q_{\text {total }}=1050$. The coupling efficiency $\eta_{c}$ was calculated by using the FDTD method to be $\sim 1 \%$. Owing to these effects, $\eta_{\text {abs }}$ is enhanced by the factor $Q_{\text {total }} \eta_{c}$ so that it actually lies between $1.3 \%$ and $2.1 \%$. But this absorption efficiency is still much lower than that for highfrequency pumping. Consequently, using the higher irradiated threshold power for resonant pumping than for $0.98 \mu \mathrm{m}$ pumping is preferable. This situation can be dramatically improved by pumping through the input waveguide, as shown schematically in Fig. 3(a). In this case, $Q_{\text {total }}=\left(Q_{a}^{-1}+Q_{\text {ver }}^{-1}\right.$ $\left.+Q_{c}^{-1}+Q_{\text {par }}^{-1}\right)^{-1}$, where $Q_{c}$ is determined by the coupling efficiency $\eta_{c}$ between the cavity and the waveguide. According to coupled mode theory, ${ }^{14}$ the absorption efficiency for pumping through the input waveguide is $\eta_{\mathrm{abs}}=4 Q_{\mathrm{total}}^{2} / Q_{c} Q_{a}$. Figure 3(b) shows the calculated result, where $Q_{\text {ver }}$ is taken to be an unknown variable. Note that $\eta_{\text {abs }}$ has a maximum when $Q_{c}=\left(Q_{q}^{-1}+Q_{\mathrm{ver}}^{-1}+Q_{\mathrm{par}}^{-1}\right)^{-1}$. Therefore, when $Q_{\mathrm{ver}}$ is

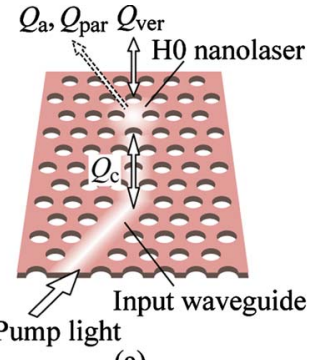

(a)

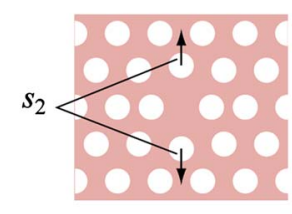

(c)

FIG. 3. (Color online) Resonant photopumping through an input waveguide. (a) Schematic of the $H 0$ nanolaser integrated with an input waveguide. (b) Plot of $\eta_{\mathrm{abs}}$ vs $Q_{c}$. Here, $Q_{a}$ and $Q_{\mathrm{par}}$ are assumed to be 2000 and 20000 , respectively, while $Q_{\text {ver }}$ is an unknown variable. (c) Graph of $Q_{\text {ver }}$ for the two cavity modes calculated with the airhole shift $s_{2}$ shown in the inset.

greater than $10^{4}$ and $Q_{c}$ is close in value to $Q_{a}, \eta_{\mathrm{abs}}$ can exceed $80 \%$. In a standard $H 0$ cavity, $Q_{\text {ver }}$ for a dipole mode is typically 2500. But this value can be enhanced by displacing two neighboring airholes in the $H 0$ cavity, as shown in Fig. 3(c). When the shift $s_{2}$ is larger than $0.08 a, Q_{\text {ver }}$ for the dipole mode increases to $10^{4}$ while that for the monopole mode remains almost constant.

Finally, we discuss the nonlinear switching behavior observed for another sample, in which the absorption efficiency was larger than that of the previous sample. Figure 4(a) shows the laser mode intensity versus the pump wavelength. A precipitous drop is observed on the long wavelength side of the peak, particularly at high pump levels. Figure 4(b) shows the laser mode intensity as a function of the irradiated power for five different pump wavelengths. When pumped at $1537.5 \mathrm{~nm}$ (slightly longer than the dipole mode), the intensity sharply increased at $P_{\text {irr }}=3.3 \mathrm{~mW}$. As $P_{\text {irr }}$ increased, the resonant pumping wavelength for the dipole mode was thermally redshifted, as shown in the inset of Fig. 4(b), while the

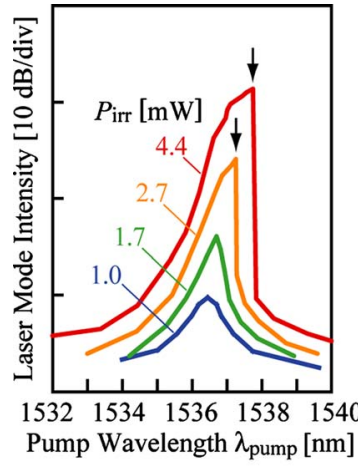

(a)

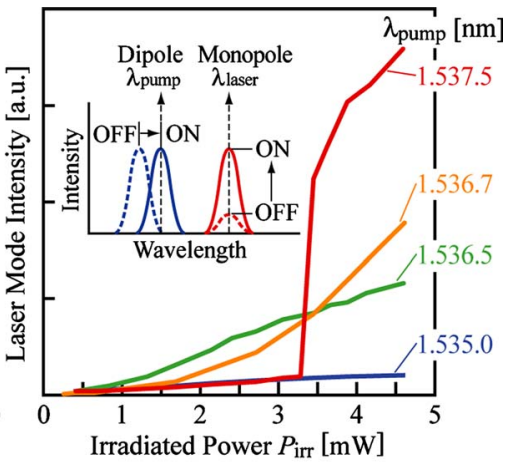

(b)
FIG. 4. (Color online) Switching behavior of the pumping wavelength and intensity in the resonant photopumping experiment. (a) Laser mode intensity vs pump wavelength for four different irradiated powers. The arrows indicate the switching wavelength. (b) Laser mode intensity vs irradiated power for five different pumping wavelengths. The inset shows the shift in the wavelength and intensity of the dipole and monopole modes, respectively. 
laser intensity of the monopole mode sharply increased. The spectral broadening shown in Fig. 4(a) can be explained by the thermal redshift and by the locking of the resonance with the upward wavelength sweep. ${ }^{15,16}$ Such characteristics suggest the possibility of bistable switching with an extinction ratio of $12 \mathrm{~dB}$. This value is higher than that for the bistability observed in passive PC cavities. ${ }^{16,17}$ It is attributed to the active operation of the laser, where switching between spontaneous and stimulated emission occurs with a change in the absorption efficiency.

In conclusion, we have demonstrated RT cw operation of a PC nanolaser by resonant optical pumping. The intensity of the monopole mode, with a modal volume of merely $0.019 \mu \mathrm{m}^{3}$, was enhanced by up to $35 \mathrm{~dB}$ above the background level when the pump wavelength was fixed at that of the dipole mode. Its high threshold is due to the low pump efficiency, but can be reduced by in-plane coupling of the pump light through the waveguide. We also observed thermal nonlinear switching with an extinction ratio of $12 \mathrm{~dB}$, demonstrating the possibility of bistable operation. We have already realized the integration of the active nanolaser with passive waveguides using a butt-joint regrowth technique. ${ }^{18,19}$ It allows resonant pumping through the input waveguide and light extraction through the output waveguide. This will enable various functionalities such as an optical 2R (reamplifying and reshaping) device or a wavelength converter.

This study was supported by a Grant-in-Aid and by a Research Fellowship of the Japanese Society for the Promotion of Science.
${ }^{1}$ O. Painter, R. K. Lee, A. Scherer, A. Yariv, J. D. O’Brien, P. D. Dapkus, and I. Kim, Science 284, 1819 (1999).

${ }^{2}$ H. Y. Ryu, S. H. Kim, H. G. Park, J. K. Hwang, Y. H. Lee, and J. S. Kim, Appl. Phys. Lett. 80, 3883 (2002).

${ }^{3}$ T. Baba, D. Sano, K. Nozaki, K. Inoshita, Y. Kuroki, and F. Koyama, Appl. Phys. Lett. 85, 3989 (2004).

${ }^{4}$ T. Yoshie, A. Scherer, J. Hendrickson, G. Khitrova, H. M. Gibbs, G. Rupper, C. Ell, O. B. Shchekin, and D. G. Deppe, Nature (London) 432, 200 (2004).

${ }^{5}$ K. Nozaki and T. Baba, Appl. Phys. Lett. 88, 211101 (2006).

${ }^{6}$ K. Nozaki, S. Kita, and T. Baba, Opt. Express 15, 7506 (2007).

${ }^{7}$ K. Nozaki, S. Kita, and T. Baba, IEEE International Semiconductor Laser Conference, Hawaii, 2006 (unpublished), Paper No. PD 1-2.

${ }^{8}$ M. Nomura, S. Iwamoto, M. Nishioka, S. Ishida, and Y. Arakawa, Appl. Phys. Lett. 89, 161111 (2006).

${ }^{9}$ T. Ide, J. Hashimoto, K. Nozaki, E. Mizuta, and T. Baba, Jpn. J. Appl. Phys., Part 2 45, L102 (2006).

${ }^{10} \mathrm{H}$. Watanabe and T. Baba, International Symposium on Compound Semiconductors, Kyoto, 2007 (unpublished), Paper No. ThC-P10.

${ }^{11}$ K. Nozaki, A. Nakagawa, D. Sano, and T. Baba, IEEE J. Sel. Top. Quantum Electron. 9, 1355 (2003).

${ }^{12}$ T. Arakawa, K. Tada, N. Kurosawa, and J. H. Noh, Jpn. J. Appl. Phys., Part 1 39, 6329 (2000).

${ }^{13}$ W. J. Choi and J. C. Yi, J. Korean Phys. Soc. 46, 1452 (2005).

${ }^{14}$ C. Manolatou, M. J. Khan, S. Fan, P. R. Villeneuve, H. A. Haus, and J. D. Joannopoulos, IEEE J. Quantum Electron. 35, 1322 (1999).

${ }^{15}$ P. E. Barclay, K. Srinivasa, and O. Painter, Opt. Express 13, 801 (2005).

${ }^{16}$ M. Notomi, A. Shinya, S. Mitsugi, G. Kira, E. Kuramochi, and T. Tanabe, Opt. Express 13, 2678 (2005).

${ }^{17}$ M. K. Kim, I. K. Hwang, S. H. Kim, H. J. Chang, and Y. H. Lee, Appl. Phys. Lett. 90, 161118 (2007).

${ }^{18} \mathrm{H}$. Watanabe and T. Baba, Electron. Lett. 42, 695 (2006).

${ }^{19}$ K. Nozaki, S. Kita, and T. Baba, International Symposium on Compound Semiconductors, Kyoto, 2007 (unpublished), Paper No. ThC-P8. 